\title{
Synthesis of 1,2-Diazepino[3,4-b]quinoxalines by 1,3-Dipolar Cycloaddition Reaction and Their Ring Transformation to Pyridazino $[3,4-b]$ quinoxalines
}

\author{
Ho Sik Kim," Seong Uk Lee, Hyoung Choul Lee, and Yoshihisa Kurasawa* \\ Department of Chemistry, Catholic Liniversity of Taegu, Glongsan 712-702, Korea \\ ${ }^{\dagger}$ School of Phamacetitical Sciences, Kitasato Lniversitv, Shirokane, Hinato-ku, Tokvo 108-86+1, Japan \\ Received November 19, 2001
}

Keywords : 1.2-Diazepino[3.4-b]quinoxalines. 1.3-Dipolar cycloaddition reaction. Ring transformation. Py ridazino[3.4-b]quinoxalines. Antibacterial activity

Fused heterocyclic systems containing a quinoxaline ring were largely investigated because they were effective in pharmacological and agrochemical areas. 1,2

In previous papers ${ }^{3-8}$ we reported the sy'nthesis of the 1.2diazepino[3.4-b]quinoxaline-5-carbonitriles 3a-e from the quinoxaline $\mathrm{N}$-oxide 1 wa the hydrazones $2 \mathrm{a}$-e and then the oxidative ring transformation of $3 \mathrm{a}-\mathrm{c}$ with $N$-bromosuccinimide/water or selenium dioxide conveniently produced the py ridazino $[3,4-b]$ quinoxalines 4a-c. respectively. From the data of the screening test. it was found that compound 3d showed a weak antibacterial activity against Xonthomonos oryzae, but compound 3e did not show antibacterial activity. Compound $\mathfrak{A c}$ exhibited antibacterial activity against Bacilh subtilis. ${ }^{6}$

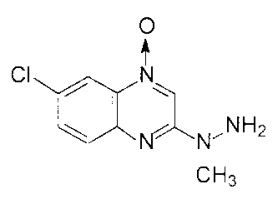

1

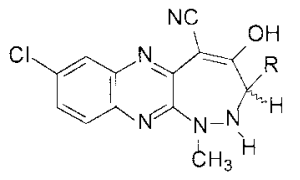

3

$\mathrm{R}: \mathbf{a}-\mathrm{C}_{6} \mathrm{H}_{4}-4-\mathrm{Cl}$ b $-\mathrm{C}_{6} \mathrm{H}_{4}-4-\mathrm{Br}$ c - $\mathrm{C}_{2} \mathrm{H}_{5}$ d-2-furyl e-2-thienyl

In this note. we undertook the synthesis of 1,2-diazepino$[3,4-b]$ quinoxalines 6 possessing the $\alpha \beta$-unsaturated moieties at the 3-position from compounds 5 and the synthesis of pyridazino[3.4-b]quinoxalines 7 by the oxidative ring transformation of compounds 6 (Scheme 1). We, also. tested in vitro antibacterial activity of these compounds.

The reaction of 6-chloro-2-(1-methy lhydrazino)quinoxaline 4-oxide 1 with $\alpha \beta$-unsaturated aldehydes such as acrolein. crotonaldehyde, $t / m s$-ciunamaldehyde and 3-(2-furyl)acrolein gave 6-chloro-2-[1-nethy]-2-(vinylmethylene)hydrazino]quinoxaline t-oxide 5a, 6-chloro-2-[1-nethyl-2-(methy lvinyl-
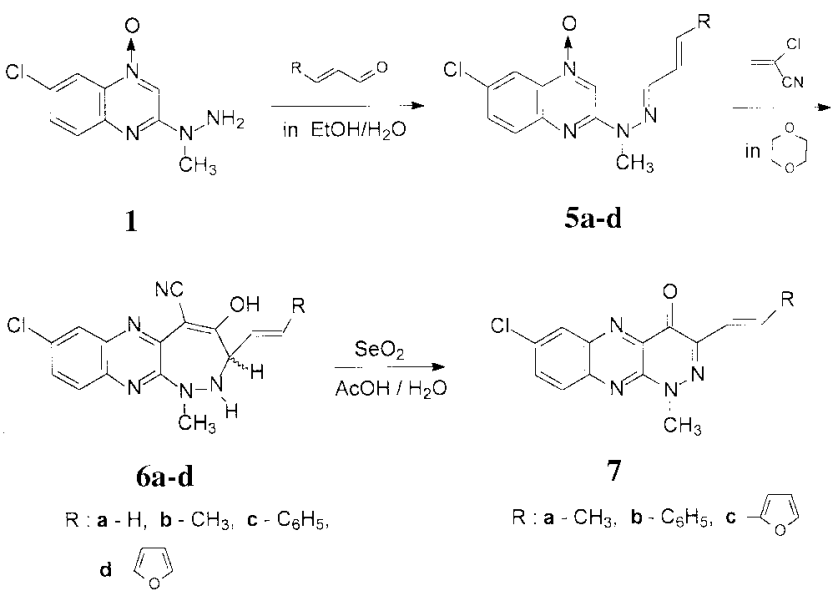

Scheme 1

methylene)hydrazino]quinoxaline 4-oxide 5b. 6-chloro-2[ I-methyl-2-(pheny lvinylmethylene)hydrazino]quinoxaline 4-oxide $5 \mathrm{c}$ and 6-chloro-2-[(2-furylviny methylene)-1-metlylhydrazino]quinoxaline 4-oxide $\mathbf{5} d$, respectively. The reaction of compounds 5 with 2-chloroacrylonitrile afforded 6-chloro-2.3-dilyydro-4-hydroxy-1-metlyyl-3-vinyl-1 H-1,2diazepino[3,4-b]quinoxaline-5-carbonitrile 6a. 6-chloro-2.3dihydro-4-hyd droxy-1-methyl-3-(metlyylvinyl)-1 H-1.2-diazepino[3.4-b]quinoxaline-5-carbonitrile $6 \mathbf{b}$ and 6-chloro-2.3dihydro-4-hydrosy -1-methyl-3-(phenylvinyl)- $1 H$-1.2-diazepino[3.4-b]quinoxaline-5-carbonitrile $6 c$ and 6-chloro-3-(2furyl-vinyl)-2,3-dihydro-4-hydroxy-1-methyl-1 $H$-1.2-diaze-

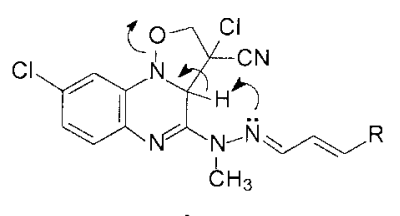

A

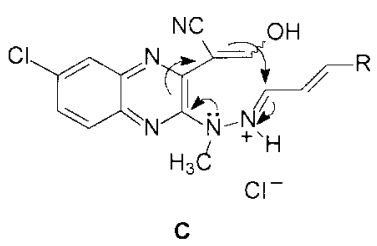

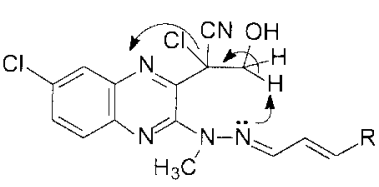

B

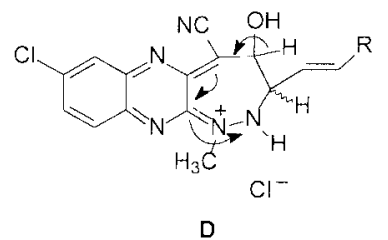


pino[3.4-b]quinoxaline-5-carbonitrile 6d, respectively. presumably wa intermediates A-D. ${ }^{3,4,6}$

The reaction of the 1.2-diazepino[3,4-b]quinoxalines 6 with selenium dioxide in acetic acid/water resulted in oxidative ring transformation to provide 7-chloro-1-methyl3-(methylvinyl)-4-oxo-1.4-dihydropyridazino [3,4-b]quinoxaline 7a. 7-chloro-1-methyl-3-(phenylvinyl)-4-oxo-1,4-dilydropyridazino[3.4-b] quinoxaline $7 \mathrm{~b}$ and 7-chloro-3-(2-furylvinyl)-1-methyl-4-oxo-1.4-dihydropyridazino[3,4-b]quinoxaline 7c. respectively. presumably via intermediates $\mathbf{E}-\mathbf{H} . ; 0$<smiles>[R]C=CC1NN(C)C2=Nc3ccc(Cl)cc3NC2=C1O</smiles>

$E$<smiles>[R]C=C[C@]1(O)C(=O)c2nc3cc(Cl)ccc3nc2N(C)N1C</smiles>

G
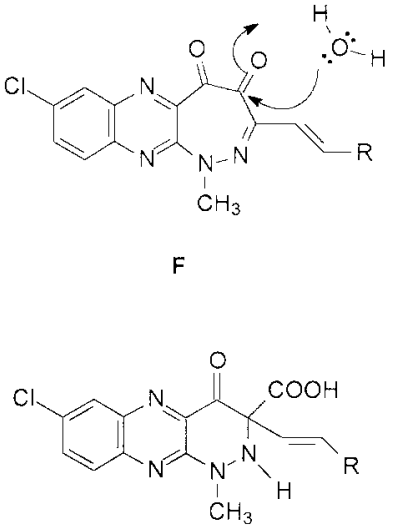

We transformed compound $\mathbf{1}$ into the hydrazone $\mathbf{8}$ so as to synthesize new condensed quinoxaline $\mathbf{1 0}$ by 1.3-dipolar cycloaddition reaction and an intramolecular alcoholysis. ${ }^{7,10}$ The reaction of compound 1 with 2.3-O-isopropylidene-Dglyceraldehyde ${ }^{11,12}$ gave 6-chloro-2-[1-methyl-2-[4-(2.2-dimethyl-1.3-dioxolanylmethylene)] hydrazino]quinoxaline 4 oxide 8 (Scheme 2). Efforts to obtain compound 9 and $\mathbf{1 0}$ from the reaction of compound 8 with 2-chloroacrylonitrile were unsuccessful.

The structure of new compounds 6 and 7 was supported by the spectral and analytical data. The 2.3-dihydro-4-hydro$\mathrm{xy}$ form of compounds 6 have already been clarified by the ineasurement of the NOE between the $\mathrm{N}_{2}-\mathrm{H}$ and $\mathrm{C}_{3}-\mathrm{H}$ protons in previous papers. ${ }^{3 .+}$

All the compounds (6 and 7) were tested for their antibacterial activity following paper disc method ${ }^{13}$ against

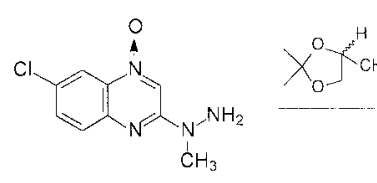

1

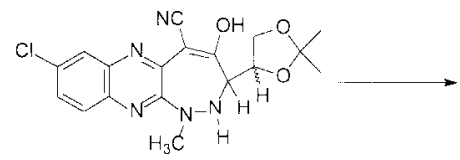

9

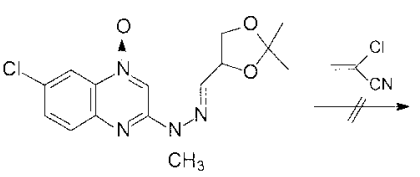

8

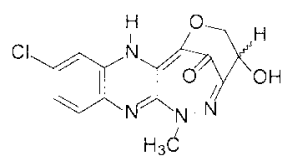

10
Scheme 2
Table $1 . I n$ Fitro antibacterial activity of the compounds

\begin{tabular}{|c|c|c|c|c|c|c|c|c|}
\hline Strains & Compounds & $6 a$ & $6 \mathrm{~b}$ & $6 \mathrm{c}$ & 6d & $7 a$ & $7 b$ & $7 c$ \\
\hline \multirow{3}{*}{$\begin{array}{l}\text { Gram-positive } \\
\text { bacteria }\end{array}$} & L. nonocvtogenes & $14^{\sigma}$ & 15 & 15 & 15 & 15 & 12 & 12 \\
\hline & S. atoweus & 15 & 12 & 13 & 13 & 14 & 12 & 11 \\
\hline & B. centus & 12 & 11 & 13 & 13 & 16 & 9 & 13 \\
\hline \multicolumn{2}{|c|}{ Gram-negative $E$. coli } & 11 & 12 & 11 & 15 & 12 & 11 & 12 \\
\hline \multirow[t]{2}{*}{ bacteriá } & S. tophimminm & 13 & 15 & 11 & 13 & 14 & 14 & 12 \\
\hline & P. fluorescens & 12 & 12 & 12 & 14 & 13 & 12 & 13 \\
\hline
\end{tabular}

Diameter of inhibition zone (mm)

Lister monocytogens ATCC 1911, Staphyococcus curets ATCC 29737. Bacillis cerets ATCC 21366, Escherichia coli ATCC 11775. Satmonella tvphimuritum ATCC 29737 and Pseudomonas fitorescens ATCC 21541. Paper disc were placed on the Tryptic soy agar spreaded with each bacteria. The plates were incubated at $37^{\circ} \mathrm{C}$ for 24 lurs. The activity was recorded by measuring the diameter of inhibition zones in $\mathrm{mm}^{1+, 15}$ and results obtained are shown in Table 1. All the compounds showed inhibitory effect against tested bacteria.

\section{Experimental Section}

All melting points were determined on a Haake Buchler melting point apparatus and are uncorrected. The ir spectra (potassium bromide) were recorded with a Mattson Polaris FT/RR spectrophotometer. The nmir spectra were measured with a Varian Gemini-200 spectrometer at $200 \mathrm{MHz}$. The chemical shifts are given in the $\delta$ scale. The mass spectra (ms) were deternined with a shimadzu GC/MS QP-5050 spectrometer. Elemental analyses were performed on an Elementar Vario EL instrument.

General procedure for the preparation of the quinoxaline 4-0xides (5a-d)

To a stirred and ice cooled suspension of compound 1 ( $\mathrm{lg}$, $4.45 \mathrm{mmol})$ and ethanol $(30 \mathrm{~mL}) /$ water $(10 \mathrm{~mL})$ was added dropwise the appropriate $\alpha, \beta$-unsaturated aldeliydes (5.34 munol, 1,2-fold molar amount) and concentrated sulfuric acid $(4 \mathrm{~mL}$ ). The reaction mixture was stirred at room temperature for 16 hours under nitrogen to precipitate yellow crystals. which were collected by suction filtration. Washing with ethanol and then $n$-hexane gave an analytically pure samples.

6-Chloro-2-[1-methyl-2-(vinylmethylene)hydrazino]quinoxaline +-Oxide (5a). Yield $60 \%$. mp $154-156{ }^{\circ} \mathrm{C}$; IR(KBr): $3086,1577,1536,1491,1386,1221,1096 \mathrm{~cm}^{-1}$; MS: $\mathrm{m} / \mathrm{z} 262\left(\mathrm{M}^{-}\right), 264\left(\mathrm{M}^{+}+2\right) ;{ }^{1} \mathrm{H}$ NMR (DMSO-d $\left.\mathrm{d}_{6}\right): 8.75$ (s, IH. C. $-\mathrm{H}), 8.23(\mathrm{~s}, \mathrm{H} . \mathrm{C}-\mathrm{H}), 7.80-7.74\left(\mathrm{~m}, 3 \mathrm{H} . \mathrm{C}_{7}-\mathrm{H}\right.$, $\mathrm{C}_{8}-\mathrm{H}$ and hydrazone $\mathrm{CH}$ ) $6.75-6.52\left(\mathrm{~m} . \mathrm{HH} . \mathrm{N}=\mathrm{CH}-\mathrm{CH}=\mathrm{CH}_{2}\right)$. 5.82 .5 .60 (m. $2 \mathrm{H}, \mathrm{N}=\mathrm{CH}-\mathrm{CH}=\mathrm{CH} \cdot 3.59$ (s. $3 \mathrm{H} . \mathrm{N}-\mathrm{CH}_{3}$ ). Anal. calcd. for $\mathrm{C}_{12} \mathrm{H}_{11} \mathrm{ClN}_{4} \mathrm{O}: \mathrm{C}, 54.87 ; \mathrm{H}, 4.22$ : N. 21.33 . Found: C, 54.76: H, 4.23; N. 21.28

6-Chloro-2-[1-methyl-2-(methylvinylmethylene)hydrazinolquinoxaline 4-Oxide (5b). Yield $88 \%$, mp 207-209: IR 
(KBr): 3088. 1577. 1536. 1485. $1098 \mathrm{~cm}^{-1}: \mathrm{MS}: \mathrm{nl} / \mathrm{z} 276$ $\left(\mathrm{M}^{+}\right), 278\left(\mathrm{M}^{+}+2\right):{ }^{1} \mathrm{H}$ NMR (DMSO-d 6$): 8.73\left(\mathrm{~s}, 1 \mathrm{H} . \mathrm{C}_{3}-\mathrm{H}\right)$. $8.24\left(\mathrm{~s}, 1 \mathrm{H}, \mathrm{C}_{5}-\mathrm{H}\right), 7.85-7.62$ (nt. $3 \mathrm{H} . \mathrm{C}_{7}-\mathrm{H} . \mathrm{C}_{s}-\mathrm{H}$ and hydrazone $\mathrm{CH}$ ), 6.48-6.15 (nt. $2 \mathrm{H}, \underline{\mathrm{CH}}=\mathrm{CHCH}_{3}$ ). 3.57 (s. $\left.3 \mathrm{H}, \mathrm{N}-\mathrm{CH}_{3}\right), 1.88\left(\mathrm{~d}, J=5.5 \mathrm{~Hz}, 3 \mathrm{H}, \mathrm{CH}_{3}\right)$. Anal. calcd. for $\mathrm{C}_{13} \mathrm{H}_{13} \mathrm{ClN}_{4} \mathrm{O}:$ C. $56.43 ;$ H. 4.74; N. 20.25. Found: C. 56.53; H. $4.78 ;$ N. 20.24 .

6-Chloro-2-[1-methyl-2-(phenylvinylmethylene)hydrazino]quinoxaline +-Oxide (5c). Yield 94\%. mp 262-264 ${ }^{\circ} \mathrm{C}$ : IR (KBr): 3027. 1580, 1531. 1491, 1216. $1091 \mathrm{~cm}^{-1}$; MS: $\mathrm{m} / \mathrm{z}$ $338\left(\mathrm{M}^{+}\right), 340\left(\mathrm{M}^{+}+2\right)$ : ${ }^{1} \mathrm{H}$ NMR $\left(\mathrm{CDCl}_{3}\right): 9.02$ (s, IH. $\mathrm{C}_{3-}$ H). 8.45 (d. $J=2.2 \mathrm{~Hz}, 1 \mathrm{H} . \mathrm{C}-\mathrm{H}) .7 .80-7.29$ (n, $8 \mathrm{H} . \mathrm{C}_{7}-\mathrm{H}$. $\mathrm{C}_{S}-\mathrm{H}$. aromatic and hydrazone $\left.\mathrm{CH}\right) .7 .10-6.82(\mathrm{~m}, 2 \mathrm{H}, \underline{\mathrm{CH}}=$ CHPh). 3.68 (s. $3 \mathrm{H} . \mathrm{N}-\mathrm{CH}_{3}$ ). Anal. calcd for $\mathrm{C}_{18} \mathrm{H}_{15} \mathrm{ClN}_{4} \mathrm{O}$ : C. 63.81: H. 4.46; N. 16.54. Found: C. 63.69; H. 4.4l: N. 16.40 .

6-Chloro-2-[(2-furylvinylmethylene)-1-methylhydrazino]quinoxaline 4-Oxide (5d). Yield $97 \%$, np $247-249{ }^{\circ} \mathrm{C}$; IR (KBr): $3107,1575,1528.1488,1219,1089 \mathrm{~cm}^{-1}:$ MS: $\mathrm{m} / \mathrm{z}$ $328\left(\mathrm{M}^{+}\right), 330\left(\mathrm{M}^{+}+2\right):{ }^{1} \mathrm{H}$ NMR $\left(\mathrm{CDCl}_{3}\right): 9.00$ (s, IH. $\mathrm{C}_{3}-$ H). 8.43 (s. $1 \mathrm{H}, \mathrm{C}_{5}-\mathrm{H}$ ), 7.75-7.50 (m. $3 \mathrm{H} . \mathrm{C}_{7}-\mathrm{H}, \mathrm{C}_{5}-\mathrm{H}$ and furan $\mathrm{C}_{\leq}-\mathrm{H}$ ). 7.46 (s. IH. hydrazone $\mathrm{CH}$ ). 6.95-6.60 (nt. $2 \mathrm{H}$. $\mathrm{N}=\mathrm{CH}-\underline{\mathrm{CH}}=\underline{\mathrm{CH}}-), 6.50-6.40\left(\mathrm{~m} .2 \mathrm{H}\right.$, furan $\mathrm{C}_{3}-\mathrm{H}$ and $\left.\mathrm{C}_{4}-\mathrm{H}\right)$. $3.66\left(\mathrm{~s}, 3 \mathrm{H}, \mathrm{N}-\mathrm{CH}_{3}\right)$. Anal. calcd for $\mathrm{C}_{16} \mathrm{H}_{13} \mathrm{CIN}_{4} \mathrm{O}_{2}: \mathrm{C}$. 58.46: H, 3.99; N. 17.04. Found: C. $58.38 ;$ H. 3.87: 16.88.

General procedure for the preparation of the 1,2diazepino [3,4-b]quinoxalines (6a-d)

A suspension of the appropriate contpounds 5 (3.82 numol) and 2-chloroacrylonitrile (15.28 mmol) in dioxane $(50 \mathrm{~mL})$ was refluxed in an oil bath for 2 hours. After cooling to room temperature. the precipitate was filtered off and the filtrate was evaporated in vacuo. The oily residue was crystallized from ethanol/water to reddish brown crystals. which were collected by suction filtration and then washed with water to give an analytically pure samples.

6-Chlor0-2,3-dihydro-4-hydroxy-1-methyl-3-vinyl-1 $H$ 1,2-diazepino[3,4-b]quinoxaline-5-carbonitrile (6a). Yield $63 \%$, mp 124-126 ${ }^{\circ} \mathrm{C}$; IR (KBr): 2220, 1599, 1558, 1525. $1486 \mathrm{~cm}^{-1}$; MS: $\mathrm{m} / \mathrm{z} 313\left(\mathrm{M}^{-}\right) .315\left(\mathrm{M}^{+}+2\right)$; ${ }^{1} \mathrm{H}$ NMR $\left(\mathrm{DMSO}-\mathrm{d}_{6}\right.$ ): 13.89 (brs, 1H, OH). 8.03 (s. IH. C. $-\mathrm{H}$ ), 7.58$7.30\left(\mathrm{~m}, 2 \mathrm{H}, \mathrm{C}-\mathrm{H}\right.$ and $\left.\mathrm{C}_{1 \mathrm{i}}-\mathrm{H}\right), 6.20-6.02\left(\mathrm{~m}, 2 \mathrm{H}, \mathrm{C}_{3}-\mathrm{H}\right.$ and $\underline{\mathrm{CH}}=\mathrm{CH}_{2}$ ). $5.38-5.00$ (nt. $2 \mathrm{H}, \mathrm{CH}=\mathrm{CH}_{2}$ ). 4.64 (s. $1 \mathrm{H}, \mathrm{NH}$ ). $3.23\left(5,3 \mathrm{H} . \mathrm{N}-\mathrm{CH}_{3}\right)$. Anal. calcd. for $\mathrm{C}_{15} \mathrm{H}_{1} \mathrm{ClN}_{5} \mathrm{O}: \mathrm{C}$. 57.42: H. 3.86; N, 22.32. Found: C, 57.22: H. 3.80; N, 21.97.

6-Chloro-2,3-dihydro-4-hydroxy-1-methyl-3-(methylvinyl)-1 $H$-1,2-diazepino $[3,+-b]$ quinoxaline-5-carbonitrile (6b). Yield $71 \%$, mp 118-120 ${ }^{\circ} \mathrm{C}$; IR (KBr): 2223. 1598. 1558. 1529. $1486 \mathrm{~cm}^{-1}$ : MS: $\mathrm{m} / \mathrm{z} 327\left(\mathrm{M}^{+}\right) .329\left(\mathrm{M}^{-}+2\right):{ }^{l} \mathrm{H} \mathrm{NMR}$ $\left(\mathrm{DMSO}-\mathrm{d}_{6}\right.$ ): 13.86 (brs, 1H, OH). 8.03 (s. IH. C. $-\mathrm{H}$ ), 7.58$7.32\left(\mathrm{~m}, 2 \mathrm{H}, \mathrm{C}_{1}-\mathrm{H}\right.$ and $\left.\mathrm{C}_{10}-\mathrm{H}\right), 5.96\left(\mathrm{~s} .1 \mathrm{H}, \mathrm{C}_{3}-\mathrm{H}\right), 5.90-5.38$ (m. $2 \mathrm{H}, \underline{\mathrm{CH}}=\underline{\mathrm{CHCH}}$ ), 4.57 (s. $1 \mathrm{H}, \mathrm{NH}$ ), 3.22 (s. $3 \mathrm{H}, \mathrm{N}$ $\mathrm{CH}_{3}$ ). 1.66 (d. $J=5.6 \mathrm{~Hz}, 3 \mathrm{H}, \mathrm{CH}_{3}$ ). Anal calcd. for $\mathrm{C}_{16} \mathrm{H}_{14} \mathrm{ClN}_{5} \mathrm{O}:$ C. $58.63 ;$ H. 4.31; N. 21.37. Found: C. 58.74; H. $4.21 ;$ N. 21.06 .

6-Chloro-2,3-dihydro-t-hydroxy-1-methyl-3-(phenylvinyl)-1 $H$-1,2-diazejino[3,t-b]quinoxaline-5-carbonitrile (6c). Yield $72 \%$. mp 168-170 $\mathrm{C}$ : IR (KBr): 2225. 1598. 1557.
1527. $1486 \mathrm{~cm}^{-1}$; MS: $\mathrm{m} / \mathrm{z} 389\left(\mathrm{M}^{-}\right), 391\left(\mathrm{M}^{+}+2\right)$; ${ }^{1} \mathrm{H}$ NMR (DMSO-d ) 13.92 (brs. IH. OH), 8.06 (s. IH. C. 7 -H) 7.60 $7.15\left(\mathrm{~m}, 7 \mathrm{H}, \mathrm{C}_{9}-\mathrm{H}, \mathrm{C}_{1 \mathrm{i}}-\mathrm{H}\right.$ and aromatic), 6.62-6.32 (m, $2 \mathrm{H}$, $\mathrm{C}_{3}-\mathrm{H}$ and $\left.\underline{\mathrm{CH}}=\mathrm{CHPlh}\right), 6.09(\mathrm{~d}, J=11.8 \mathrm{~Hz}, 1 \mathrm{H} . \mathrm{CH}=\underline{\mathrm{CHPh}})$, 4.81 (s, IH. NH). 3.25 (s. $3 \mathrm{H} . \mathrm{N}-\mathrm{CH}_{3}$ ). Anal. calcd. for $\mathrm{C}_{31} \mathrm{H}_{16} \mathrm{ClN}_{5} \mathrm{O}: \mathrm{C}, 64.70: \mathrm{H}, 4.14: \mathrm{N}, 17.97$. Found: C. 64.19; $\mathrm{H}, 3.98: \mathrm{N}, 17.62$.

6-Chloro-3-(2-funylvinyl)-2,3--lihydro-t-hydroxy-1-methyl1H-1,2-diazepino[3,4- $b$ ]quinoxaline-5-carbonitrile (6d). Yield 86\%, mp 132-134 ${ }^{\circ} \mathrm{C}$; IR (KBr): 2227, 1598, 1557. 1526. $1486 \mathrm{~cm}^{-1}$; MS: $\mathrm{m} / \mathrm{z} 379\left(\mathrm{M}^{-}\right), 381\left(\mathrm{M}^{+}+2\right)$; ${ }^{1} \mathrm{H}$ NMR

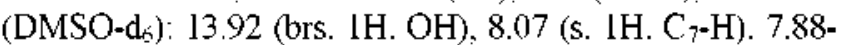
7.32 (m. $3 \mathrm{H}$. C. $\mathrm{C}_{0}-\mathrm{H}_{1} \mathrm{C}_{1 \mathrm{j}} \mathrm{H}$ and furan $\left.\mathrm{C}_{5}-\mathrm{H}\right), 6.58-6.20(\mathrm{~m}$, $4 \mathrm{H} . \mathrm{C}_{3}-\mathrm{H}, \underline{\mathrm{CH}}=\mathrm{CH}$ furan, furan $\mathrm{C}_{3}-\mathrm{H}$ and $\left.\mathrm{C}_{4}-\mathrm{H}\right) .6 .08(\mathrm{~d}, J=$ $12.0 \mathrm{~Hz}, \mathrm{lH}, \mathrm{CH}=\mathrm{CHfuran}), 4.8 \mathrm{l}$ (s. $\mathrm{lH}$. NH), $3.23(\mathrm{~s}, 3 \mathrm{H}$, $\mathrm{N}-\mathrm{CH}_{3}$ ). Anal. calcd for $\mathrm{C}_{19} \mathrm{H}_{1+} \mathrm{ClN}_{5} \mathrm{O}_{3}$ : C. 60.09: $\mathrm{H}, 3.72$ : N. 18.44. Found: C. 59.18: H, 3.57; N. 18.87.

General procedure for the preparation of the pyridazino[3,4-b]quinoxalines (7a-c)

A solution of the appropriate compounds $6(3.06 \mathrm{mmol})$ and selenium dioxide $(6.12 \mathrm{mmol})$ in acetic acid $(20 \mathrm{~mL}) /$ water $(10 \mathrm{~mL})$ was refluxed in an oil bath for 1 hour. The reaction mixture was filtered. and the filtrate was evaporation in vacuo to give brick red crystals, which were triturated with water and then collected by suction filtration Recrystallization from N.N-dimethylformamide/ethanol/ water afforded violet needles.

7-Chloro-1-methyl-3-(methylvinyl)-4-oxo-1,4-dlihydropyridazino[3,4-b]quinoxaline (7a). Yield $58 \%$, mp 174 $176{ }^{\circ} \mathrm{C}:$ IR $(\mathrm{KBr}): 1645,1537,1467 \mathrm{~cm}^{-1}$ : MS: $\mathrm{m} / \mathrm{z} 286$ $\left(\mathrm{M}^{-}\right), 288\left(\mathrm{M}^{+}+2\right):{ }^{1} \mathrm{H}$ NMR $\left(\mathrm{CDCl}_{2}\right): 8.35(\mathrm{~d}, J=1.8 \mathrm{~Hz}$, IH. $\left.\mathrm{C}_{6}-\mathrm{H}\right) .8 .02$ (d, $\left.J=9.4 \mathrm{~Hz} .1 \mathrm{H} . \mathrm{C}_{9}-\mathrm{H}\right), 7.83$ (dd, $J=2.1$, $9.0 \mathrm{~Hz} . \mathrm{IH} . \mathrm{C} \cdot \mathrm{H}), 7.22-7.02$ (m. lH. $\left.\mathrm{CH}=\mathrm{CHCH}_{3}\right), 6.79(\mathrm{~d}$, $\left.J=15.8 \mathrm{~Hz}, 1 \mathrm{H}, \underline{\mathrm{CH}}=\mathrm{CHCH}_{3}\right) .4 .26\left(\mathrm{~s}, 3 \mathrm{H} . \mathrm{N} \cdot \mathrm{CH}_{3}\right), 1.99(\mathrm{~d}$, $\left.J=6.7 \mathrm{~Hz}, 3 \mathrm{H} . \mathrm{CH}_{2}\right)$. Anal. calcd. for $\mathrm{C}_{14} \mathrm{H}_{11} \mathrm{ClN}_{4} \mathrm{O}: \mathrm{C}$. 58.65: H. 3.87; N. 19.54. Found: C. 58.33: H. 3.72; N. 19.32

7-Chloro-1-methyl-3-(phenylvinyl)-4-0x0-1,4-dihydropyridazino[3,4-b]quinoxaline (7b). Yield 56\%, mp $273-$ $275^{\circ} \mathrm{C}$ : IR (KBr): 1632, 1536, l461 cm- MS: $\mathrm{cm} / \mathrm{z} 348$ $\left(\mathrm{M}^{-}\right), 350\left(\mathrm{M}^{+}+2\right):{ }^{l} \mathrm{H}$ NMR $\left(\mathrm{CDCl}_{2}\right): 8.37(\mathrm{~d}, J=2.2 \mathrm{~Hz}$, $\left.\mathrm{IH}, \mathrm{C}_{6}-\mathrm{H}\right) .8 .06-7.28\left(\mathrm{~m}, 9 \mathrm{H} \mathrm{C} \mathrm{C}_{8}-\mathrm{H} . \mathrm{C}-\mathrm{H}\right.$. aromatic and vinylic H), $4.33\left(\mathrm{~s}, 3 \mathrm{H}\right.$. N-CH ). Anal. calcd. for $\mathrm{C}_{10} \mathrm{H}_{12} \mathrm{ClN}_{4} \mathrm{O}: \mathrm{C}$, 65.43: H, 3.76; N. 16.06. Found: C. 65.57: H. 3.62; N. 15.87

7-Chloro-3-(2-furylvinyl)-1-methyl-4-oxo-1,4-dihydropyridazino[3,4-b]quinoxaline (7c). Yield $71 \%$. mp $257-$ $259^{\circ} \mathrm{C}$ : IR (KBr): 1644, 1535, $1462 \mathrm{~cm}^{-1}$ : MS: $\mathrm{m} / \mathrm{z} 338$ $\left(\mathrm{M}^{-}\right), 340\left(\mathrm{M}^{+}+2\right):{ }^{l} \mathrm{H}$ NMR $\left(\mathrm{CDCl}_{2}\right): 8.33(\mathrm{~d}, J=2.1 \mathrm{~Hz}$, 1H. C. $-\mathrm{H}), 8.02$ (d. $J=9.1 \mathrm{~Hz}, 1 \mathrm{H} . \mathrm{C}-\mathrm{H}), 7.92-7.72$ (m. $2 \mathrm{H}$,

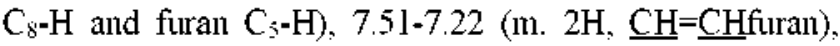
$6.52(\mathrm{~d}, J=3.3 \mathrm{~Hz}$. $1 \mathrm{H}$. furan $\mathrm{C}, \mathrm{H}), 6.45$ (dd, $J=1.5 .3 .2$ $\mathrm{Hz}$. $1 \mathrm{H}$, furan $\left.\mathrm{C}_{4}-\mathrm{H}\right), 4.31\left(\mathrm{~s}, 3 \mathrm{H} . \mathrm{N}-\mathrm{CH}_{3}\right)$. Anal. calcd for $\mathrm{C}_{17} \mathrm{H}_{11} \mathrm{ClN}_{4} \mathrm{O}_{2}:$ C. $60.28:$ H. 3.27; N, 16.54. Found: $\mathrm{C}$. 60.12: H. 3.38: N, 16.37.

6-Chloro-2-[1-methyl-2-[4-(2,2-(limethy]-1,3-dioxolanylmethylene)]hydrazino]quinoxaline t-Oxide (8). A suspension of compound 1 ( $10 \mathrm{~g}, 44.5 \mathrm{mmol}$ ) and 2.3-O. isopropylidene-D-glyceraldehy de $(8.7 \mathrm{~g} .66 .9 \mathrm{mmol})$ in dry 
benzene $(300 \mathrm{~mL})$ was refluxed on a boiling water bath for 4 hours to give a clear solution. Evaporation of the solvent in worto gave yellow crystals, which were collected by suction filtration and washed with ethanol and then $n$-hexane to give an analytically pure sample $(8.12 \mathrm{~g})$. Evaporation of the solvent in wicho afforded yellow crystals of compound 8. which were collected by suction filtration and washed with ethanol $(5.51 \mathrm{~g})$, total yield, $13.63 \mathrm{~g}(91 \%)$

Compound 8 had mp 153-155 ${ }^{\circ} \mathrm{C}$; IR (KBr) 3073. 2922. $1575,1540,1486,1402,1227.1069 \mathrm{~cm}^{-1}:$ MS: $\mathrm{m} / \mathrm{z} 336$ $\left(\mathrm{M}^{+}\right), 338\left(\mathrm{M}^{+}+2\right):{ }^{1} \mathrm{H}$ NMR (DMSO-d $\left.\mathrm{d}_{6}\right): 8.76$ (s, lH. $\mathrm{C}_{3}-\mathrm{H}$ ). $8.26\left(\mathrm{~s}, 1 \mathrm{H} . \mathrm{C}_{5}-\mathrm{H}\right) .7 .80\left(\mathrm{~s}, 2 \mathrm{H}, \mathrm{C}_{7}-\mathrm{H}\right.$ and $\left.\mathrm{C}_{8}-\mathrm{H}\right) .7 .22(\mathrm{~d} . J=$ $6.1 \mathrm{~Hz}$. $\mathrm{IH}$. hydrazone $\mathrm{CH}$ ) 4.79 (q. $J=6.3 \mathrm{~Hz}$, dioxolane $\left.\mathrm{C}_{4}-\mathrm{H}\right)$. 4.26-3.92 (m, 2H. dioxolane $\left.\mathrm{C}_{\hat{\Sigma}}-\mathrm{H}\right) .3 .56(\mathrm{~s}, 3 \mathrm{H} . \mathrm{N}-$ $\mathrm{CH}_{3}$ ). $\mathrm{I} .42\left(\mathrm{~s}, 3 \mathrm{H}\right.$. dioxolane $\mathrm{C}_{2}-\mathrm{CH}_{3}$ ), 1.36 (s. $3 \mathrm{H}$, dioxolane $\mathrm{C}-\mathrm{CH}_{3}$ ).

Acknowledgment. This work was supported by Korea Research Foundation Grant (KRF- 99-015-DP0265).

\section{References}

1. Cheeseman. C. W. H.: Cookson. R. F. The Chemishy of Heterocyctic Contpounds. Condensed Purazines: Weissberger. A.. Taylor. E. C.. Eds.: John Wiley and Sons: New York. 1979: pp 1-290.
2. Sato. N. Comprohensive Heterocyclic Chemistry: Katritzhy, A. R.. Rees. C. W.. Scriven. E. F.. Eds.: Pergamon Press: Osford. U. K.. 1996: Vol. 6. pp 234-278.

3. Kiml. H. S.: Kurasawa. Y.: Yoshii. C.: Masuyama. M.: Takada. A.: Okamoto. Y. J. Heterocvelic Chem. 1990, 27,819.

4. Kim, H. S.: Kurasawa, Y: Yoshii. C.: Masuyama, M.: Takada. A.: Okamoto. Y. J. Heterocychic Chem 1990, 27, 2197,

5. Kurasawa. Y.: Kim. H. S.: Kawano. T.: Katoh. R.: Takada. A.: Okamoto. Y. J. Heterocyclic Chent. 1991. 28. 199.

6. Kurasawa. Y: Ohshima. S.: Kishimoto. Y.: Ogura. M.: Okamoto. Y.: Kim, H. S. Heterocycles 2001. 5t, 359 .

7. Kurasawa. Y; Kureyama. T; Yoshishiba. N.; Katoh, R.: Takada, A.: Kim. H. S.: Okamoto, Y. J. Heterocyclic Chem. 1993. 30, 537.

8. Kim. H. S.: Teong. G.: Lee. H. C.: Kim. J. H.: Park. Y. T.: Ohamoto. Y.: Kajiwara. S.: Kurasawa. Y. J. Heterocwlic Chem. 2000. 37. 1277.

9. Kurasawa. Y: Kim, H. S:- Katoh, R:- Kawano, T.: Takada, A.; Okamoto. Y.J. Heterocychic Chem, 1990, 27, 2269.

10. Kurasawa. Y: Kim, H. S.; Katoh, R.; Kawano, T.: Takada, A.; Okamoto. Y. J. Heterocyclic Chen 1991. 28.787.

11. Baer. E.: Fischer. H. O. L. J. Biological Chem. 1939. 128. 463.

12. Horton. D.: Hughes. J. B.: Thomson. J. K. J. Org. Chem. 1968. 33. 728 .

13. Bauer, A. W.: Kibby, M. M.: Sherris, J. C. Tuck. M. Am. J. Clm. Pathol. 1966, 45.493.

14. Pulusani. S. R.: Rao. D. R.: Sunki. G. R. J. Food Sci. 1979. 4 . 575.

15. Conner. D. E.: Beuchat. L. R. J. Food Sci. 1984. 49.429. 